Eur. J. Clin. Chem. Clin. Biochem.

Vol. 32, 1994, pp. 583-587

(C) 1994 Walter de Gruyter \& Co.

Berlin · New York

\title{
Vitamin E Compared with Other Potential Risk Factor Concentrations in Patients with and without Coronary Artery Disease:
}

\section{A Case-Matched Study}

\author{
By F. Van Lente, Rose Daher and Joan A. Waletzky
}

Section of Biochemistry, Division of Pathology and Laboratory Medicine, Cleveland Clinic Foundation, Cleveland, Ohio, U.S.A.

(Received February 21/March 24, 1994)

Summary: Thirty six individuals with angiographic evidence of coronary atherosclerosis and thirty six individuals without coronary disease, matched for a variety of cardiovascular risk factors including age, sex, smoking, hypertension, diabetes and family history, were evaluated for their serum concentrations of vitamin $E$, total cholesterol, triacyglycerols, high density lipoprotein-cholesterol, low density lipoprotein-cholesterol, apolipoprotein A-I, and apolipoprotein B. Apolipoprotein B, low density lipoprotein-cholesterol and total cholesterol concentrations were unequivocally higher in patients with coronary artery disease. Triacylglycerols were marginally higher in patients with disease. The antioxidant vitamin E ( $\alpha$-tocopherol) was significantly higher in patients with atherosclerosis when compared with controls $(35.1 \pm 17.0 \mu \mathrm{mol} / 1$ vs. $29.0 \pm 13.2 \mu \mathrm{mol} / 1, \mathrm{p}=0.017)$. However, $\alpha$-tocopherol concentrations were strongly associated with lipid concentrations and normalization to the total cholesterol concentrations produced ratios which were not significantly different in the two groups. Logistic regression analysis revealed that the association of lipid risk factors with coronary stenosis was determined primarily by the difference in total cholesterol values. This study demonstrated that in this group of patients referred for angiography and matched for other risk factors, higher $\alpha$-tocopherol concentrations were associated with patients with coronary disease and were not useful for assessing risk of coronary artery disease.

\section{Introduction}

Major longitudinal studies have established the unequivocal relationship of serum lipoproteins to the risk for coronary artery disease. These findings have been corroborated by several therapeutic intervention studies and wealth of basic research. This, in turn, has led to a national initiative in The United States to standardize the measurement of serum cholesterol and to reduce its concentration in the population at large by risk factor modification.

There is also significant current interest in the role of free radical metabolism in the pathogenesis of atherosclerosis $(1,2)$. This is supported, in part, by evidence that low density lipoproteins are susceptible for free radical-induced oxidation in vitro and these altered particles are taken up by macrophages and internalized resulting in the formation of foam cells (1). The latter cells are well-known constituents of atherosclerotic plaque. It follows, therefore, that determinants of oxidation in the circulation might directly affect the steady state oxidative state of low density lipoproteins. This possibility has prompted studies of the role of oxidants and antioxidants such as vitamin $E$ ( $\alpha$-tocopherol) in the genesis of coronary artery disease.

We chose to evaluate the value of direct measurement of the antioxidant, $\alpha$-tocopherol in a group of patients referred for coronary angiography. The established lipid and lipoprotein constituents were analyzed for comparison of their strength of association with coronary artery disease. In an effort to minimize other confounding variables, each patient found to be without coronary artery disease was matched with another who did have disease 
with regard to other, pertinent risk factors including age, sex, family history of heart disease, hypertension and smoking. In this manner, we hoped to emphasize the relationship of the laboratory results to the presence or absence of disease.

\section{Materials and Methods}

Patients

Three hundred and seventy seven patients referred to the Cleveland Clinic Foundation for coronary angiography were considered for the study. Patients participating in this study signed appropriate informed consent approved by the Institutional Review Board. Coronary artery disease was considered present if greater than $30 \%$ of a major vessel was stenosed and the final diagnosis was coronary artery disease. Coronary artery disease was considered absent only if there was no defined stenosis in all vessels. Of this group, 43 males were found to be free of disease and the remaining group of positive patients was evaluated for optimal match of age, sex, smoking history, hypertension, angina, diabetes mellitus, ECG abnormalities, past myocardial infarction, and family history as obtained from the medical record. As a result of this process and the availability of sufficient sample, 36 pairs of matched cases were included for'study for a total of 72 individuals.

\section{Sampling}

Serum was obtained at the time of admission for cardiac catheterization, aliquoted and stored frozen at $-70^{\circ} \mathrm{C}$ until time of analysis except for total cholesterol, triacylglycerols and high density lipoprotein-cholesterol which were determined the day of sample collection. All patients had fasted for at least 12 hours before blood sampling. Whenever possible, the number of separate analytical runs was minimized.

\section{Apolipoprotein assays}

Apolipoprotein A-I and apolipoprotein B were measured with an Array nephelometric protein analyzer (Beckman Instruments, Brea CA) with reagents obtained from the manufacturer and according to their instructions.

\section{$\alpha$-Tocopherol assay}

$\alpha$-Tocopherol was measured using reverse phase high performance liquid chromatography. In brief, $0.5 \mathrm{ml}$ of plasma is combined with an equal volume of a solution of $D$ - $\alpha$-tocopherol acetate (internal standard), $25 \mathrm{mg} / \mathrm{l}$ in ethanol, mixed, $1.0 \mathrm{ml}$ of hexane is added the mixture vortexed for 45 seconds and centrifuged to separate layers. The top layer is removed, evaporated to dryness under nitrogen at room temperature and reconstituted with $200 \mu \mathrm{l}$ of mobile phase. Chromatography was carried out using a $\mathrm{C}$ - 18 reverse-phase column $(30 \mathrm{~cm}$, Millipore Waters Bondapak, Milford CT) with a C-18 guard column. System components were all obtained from Millipore Waters. The mobile phase consisted of methanol : water $(95+5$, by vol.), flow rate was $2.5 \mathrm{ml} / \mathrm{min}$ and detection was accomplished by measurement of absorbance at $280 \mathrm{~nm}$. Standardization was effected using a series of standards prepared by dissolving defined amounts of $\alpha$-tocopherol and internal standard in absolute ethanol and comparison of peak height ratios after chromatography in the usual manner. Typical retention times were 2.35 $\mathrm{min}, 8.82 \mathrm{~min}$ and $10.88 \mathrm{~min}$ for retinol, $\alpha$-tocopherol and $\alpha$-tocopherol acetate respectively.

\section{Lipid assays}

Total cholesterol was determined enzymatically with reagents obtained from Boehringer Manheim Diagnostics (Indianapolis, IN) with a Hitachi 717 analyzer according to the manufacturer's protocol. This method was standardized to the National Cholesterol Reference Standard. Triacylglycerols were measured enzymatically with reagents obtained from Boehringer; results were corrected for the presence of free glycerol by use of lipase-free blank reagent. High density lipoprotein cholesterol was measured using the dextran sulfate $\left(M_{\mathrm{r}} 50000\right)$ magnesium chloride precipitation method as described elsewhere (3). Cholestefol in high density lipoprotein subfractions were quantified according to the dextran sulfate-magnesium chloride method described elsewhere (4). Low density lipoprotein and very low density lipoprotein cholesterol concentrations were calculated from the corresponding triacylglycerols, total cholesterol and high density lipoprotein-cholesterol concentrations using the Friedewald formula if triacyglycerol values were less than $4.57 \mathrm{mmol} / \mathrm{l}(4000 \mathrm{mg} / \mathrm{l})$.

\section{Ultracentrifugation}

If triacyglycerol values exceeded $4.57 \mathrm{mmol} / \mathrm{l}$, cholesterol in lipoprotein fractions was estimated by ultracentrifugation according to a standard protocol (5). One $\mathrm{ml}$ of serum was added to $4.0 \mathrm{ml}$ of $9.0 \mathrm{~g} / \mathrm{l}$ sodium chloride (density $=1.006 \mathrm{~kg} / \mathrm{l}$ ) containing $1.0 \mathrm{~g} / \mathrm{l}$ sodium ethylenediamine tetracetate in an ultracentrifuge tube. The sample was ultracentrifuged for 15 hours at $16^{\circ} \mathrm{C}$ at $109074 \mathrm{~g}$ relative centrifugal force. The tube was subsequently placed in a tube cutter and the top $1.5 \mathrm{ml}$ fraction removed volumetrically with a $2.0 \mathrm{ml}$ plastic syringe followed by removal of the lower remaining fraction after mixing. Cholesterol in the fractions was determined as described above the very low density and low density lipoprotein-cholesterol concentrations derived after correction for recovery.

\section{Statistics}

All data distributions were evaluated for normalcy using the $\mathrm{Kol}$ mogorov-Smirnov test and non-parametric methods used where appropriate (6). The Mann-Whitney test for variance was used to compare variables in the group with and without coronary artery disease (6). Probability values of less than 0.05 were considered significant. Because very low density lipoprotein-cholesterol was calculated from triacyglycerols in the majority of subjects, this variable was considered dependent on triacyglycerols and was not treated separately. Spearman correlation coefficients were calculated (6). Associations amongst variables were assessed with stepwise multivariate linear regression (6). Association of significant variables with the presence or absence of coronary artery disease was investigated with stepwise logistic regression (7). All statistical analyses were conducted using SYSTAT software (Evanston, IL) except for logistic regression which was performed using BMDP software (Berkeley, CA).

\section{Results}

The thirty six matched cases used in this study included 10 of 36 with confirmed angina, 31 of 36 with ECG changes, 14 of 36 with hypertension, 4 of 36 diabetes, 1 of 36 with history of myocardial infarction and 8 of 36 who were currently smoking. Eighteen of 36 paired cases were age matched within one year, 13 of 36 were within $2-5$ years and 5 of 36 were within $6-8$ years. The average age of the group without coronary artery disease was $50.0 \pm 6.0$ years and the average age of the group with disease was $51.0 \pm 5.5$ years and this difference was not significant. The average body mass index [defined as weight $(\mathrm{kg})$ : height $\left(\mathrm{m}^{2}\right)$; values 
greater than 30 indicate obesity] was similar in both groups $\left(27.9 \pm 4.4 \mathrm{~kg} / \mathrm{m}^{2}, 28.1 \pm 3.7 \mathrm{~kg} / \mathrm{m}^{2}, \mathrm{p}=0.66\right.$, Mann-Whitney test). All subjects were males. Specific dietary histories were not available for these subjects but there was no evidence in the medical record that any subject was prescribed or was following a low-fat diet or had unusual dietary habits. None of the subjects were taking vitamin $\mathrm{E}$ or selenium supplements and two were taking vitamin $C$ supplements.

Table 1 lists the average analyte values found in the group without abnormalities on angiography and determined to have normal coronary arteries compared with the group with atherosclerosis. The components assayed are listed in decreasing order of significance as determined by the Mann-Whitney test. High density lipoprotein-2 exhibited slightly higher values in patients without coronary artery disease $(0.176 \pm 0.173 \mathrm{mmol} / \mathrm{l})$ than in those with coronary artery disease $(0.137$ $\pm 0.121 \mathrm{mmol} / \mathrm{l})$, but this difference was not significant ( $p=0.37$, Mann-Whitney test). Similar findings were observed for high density lipoprotein-3 $(0.778 \pm 0.132$ vs. $0.747 \pm 0.150 \mathrm{mmol} / \mathrm{l}, \mathrm{p}=0.41$ ). The mass ratios of high density lipoprotein-cholesterol and apolipoprotein A-I to apolipoprotein B did not reveal differences between groups greater than those shown in table 1 for ratio components. In most cases, the differences were less significant.

Apolipoprotein-B and low density lipoprotein-cholesterol were significantly higher in patients with coronary artery disease, a finding consistent with most other studies, but vitamin $\mathrm{E}$, generally considered a protectant, was significantly higher in this group as well. To explore this finding further, the correlations between the antioxidants $\alpha$-tocopherol and the remaining analytes were determined and these are shown in table 2. Significant correlations were obtained between $\alpha$-tocopherol and total cholesterol, triacyglycerols and the cholesterol fractions. Stepwise multivariate linear regression analysis revealed that the strongest association of those listed was between
Tab. 2 Correlation of $\alpha$-tocopherol with other analytes.

\begin{tabular}{lc}
\hline Analyte & $\begin{array}{c}\text { Correlation } \\
\text { coefficient }\end{array}$ \\
\hline Cholesterol & $0.55^{*}$ \\
Triacyglycerols & $0.64^{*}$ \\
HDL-cholesterol & $-0.41^{*}$ \\
LDL-cholesterol & $0.41^{*}$ \\
Apolipoprotein B & $0.54^{*}$ \\
Apolipoprotein A-I & -0.22 \\
\hline
\end{tabular}

$* \mathrm{p} \leq 0.01$

$\alpha$-tocopherol and low density lipoprotein-cholesterol with a multivariate $R=0.489$. The resultant linear regression equation was $\alpha$-tocopherol $=0.002$ (TRIG) +0.005 (LDL) + 0.317 .

Due to the strong association of lipophilic $\alpha$-tocopherol with lipoprotein particles, it has been suggested that it is more appropriate to express $\alpha$-tocopherol concentrations in plasma as a ratio to lipoprotein content (1). When mass ratios were calculated using $\alpha$-tocopherol and total cholesterol concentrations, the average ratio was not significantly different $(p=0.14)$ in patients without coronary artery disease $(6.2 \pm 2.9)$ compared with those with coronary artery disease $(6.6 \pm 2.7)$. The mass ratios of $\alpha$-tocopherol to triacyglycerols were not significant different $(8.5 \pm 5.1$ vs. $7.8 \pm 4.1, \mathrm{p}=0.66)$ as were the ratios of $\alpha$-tocopherol to low density lipoprotein-cholesterol $(9.3 \pm 4.3$ vs. $9.8 \pm 4.8, \mathrm{p}=0.53)$. Normalization of $\alpha$-tocopherol concentrations to apolipoprotein concentrations also did not reveal any significant differences between groups.

Logistic regression analysis was performed in a stepwise manner to further assess the association of the risk factors listed in table 3 with the presence of coronary artery disease. Total cholesterol demonstrated the strongest association with atherosclerosis, followed by triacyglycerols, low density lipoprotein-cholesterol, and $\alpha$-tocopherol. Apolipoprotein B demonstrated a weaker associ-

Tab. 1 Comparison of average (standard deviation) analyte values in patients with and without coronary artery disease.

\begin{tabular}{|c|c|c|c|c|c|c|}
\hline \multirow{3}{*}{$\begin{array}{l}\text { No. } \\
\text { Sụbjeçts }\end{array}$} & & \multicolumn{4}{|c|}{ Coronary artery disease } & \multirow[t]{3}{*}{ P* } \\
\hline & & \multicolumn{2}{|l|}{ absent } & \multicolumn{2}{|l|}{ present } & \\
\hline & & 36 & & 36 & & \\
\hline Apolipoprotein B & $\mathrm{mg} / \mathrm{l}$ & 956 & $(306)$ & 1080 & $(254)$ & 0.010 \\
\hline LDL-cholesterol & $\mathrm{mmol} / 1$ & 3.65 & $(1.00)$ & 4.15 & $(1.02)$ & 0.017 \\
\hline$\alpha$-Tocopherol & $\mu \mathrm{mol} / 1$ & 29.0 & $(13.2)$ & 35.1 & $(17.0)$ & 0.017 \\
\hline Cholesterol & $\mathrm{mmol} / \mathrm{l}$ & 5.34 & (1.11) & 5.96 & $(1.16)$ & 0.033 \\
\hline Triacyglycerols & $\mathrm{mmol} / \mathrm{l}$ & 2.05 & $(1.12)$ & 2.96 & $(2.12)$ & 0.097 \\
\hline HDL-cholesterol & $\mathrm{mmol} / 1$ & 0.95 & $(0.28)$ & 0.87 & $(0.19)$ & 0.261 \\
\hline Apolipoprotein A-I & $\mathrm{mg} / \mathrm{l}$ & 1151 & $(195)$ & 1126 & $(171)$ & 0.700 \\
\hline
\end{tabular}

* Mann-Whitney Test 
Tab. 3 Logistic regression analysis of laboratory-derived risk factors.

\begin{tabular}{llll}
\hline Analyte & $\begin{array}{l}\text { Log }^{\mathrm{a}} \\
\text { likelihood }\end{array}$ & $\begin{array}{l}\mathrm{p}^{\mathrm{a}} \\
\text { value }\end{array}$ & $\begin{array}{l}\mathrm{p}^{\mathrm{b}} \\
\text { value }\end{array}$ \\
\hline Cholesterol & -47.29 & 0.022 & 0.022 \\
Triacylglycerols & -47.32 & 0.028 & 0.144 \\
LDL-cholesterol & -47.69 & 0.035 & 0.777 \\
$\alpha$-Tocopherol & -48.47 & 0.090 & 0.400 \\
Apolipoprotein B & -48.95 & 0.167 & 0.493 \\
\hline
\end{tabular}

a First step with only the constant term included in model

b Second step with cholesterol included in model

ation. However, when cholesterol was included in the categorical regression model as a predictor, the independent association of the other risk factors was diminished to insignificance. Therefore, the association of these factors with atherosclerosis in this study group is accounted for by the difference in total cholesterol.

\section{Discussion}

The majority of patients who underwent angiography included in this study were referred for catheterization due to either possible cardiac-related chest pain or abnormal electrocardiograms. The usual lipid values, total cholesterol and triacylglycerols, were known to the referring physician and were usually noted in the review of medical history at the time of angioplasty. Therefore, a subtle selection bias relative to these analyte concentrations cannot be totally ruled out although the average total cholesterol value found in patients without angiography evidence of stenosis was lower than a mean of 5.75 $\pm 1.77 \mathrm{mmol} / \mathrm{l}$ observed in 5800 male outpatients seen at our facility for health assessment. It is of interest that the average high density lipoprotein-cholesterol value in patients without coronary disease was relatively low, even when compared with our own mean for 5800 male outpatients $(1.13 \pm 0.31 \mathrm{mmol} / \mathrm{l})$. The significance of this finding remains unclear. High density lipoproteincholesterol was without exception not referenced in the review of patient history and reasons for referral, so although selection bias cannot be ruled out, there is no compelling evidence that it was present with respect to decreased high density lipoprotein-cholesterol values. All patients with hypertension and on medication were case-controlled such that the overall effect of drugs such as beta-blockers known to lower high density lipoprotein should be minimal.

$\alpha$-Tocopherol demonstrated a clear significant difference between groups. The difference in $\alpha$-tocopherol concentration is likely due to its association with lipoproteins, as evidenced by the correlations shown in table 2. This leads to significantly higher $\alpha$-tocopherol concentrations in individuals with higher average lipid concentrations, a somewhat counterintuitive finding. Normalization of $\alpha$-tocopherol concentrations to cholesterol, in particular, eliminated the significance of the difference between groups, although this ratio tended to be higher in patients without coronary stenosis. A higher ratio of vitamin $\mathrm{E}$ to low density lipoprotein-cholesterol, expressed variously on a mass-to-molar basis, or a mass-to-mass basis or on a molar-to-molar basis had been suggested to reduce risk of coronary atherosclerosis regardless of cholesterol concentrations $(1,8)$. This ratio can also be expressed in terms of apolipoprotein B or lipoprotein protein content. However, we did not observe an increased significance in average values between groups when $\alpha$-tocopherol was expressed as a ratio to apolipoprotein B rather than to low density lipoprotein-cholesterol.

$\alpha$-Tocopherol is, on a molar basis, the major antioxidant associated with low density lipoprotein (1). Other antioxidants such as carotenoids, ubiquinol-10 and hydrophilic antioxidants such as ascorbic acid are present at much lower concentrations (1). However, the presence of a given concentration of an antioxidant such as $\alpha$ tocopherol may not accurately reflect the anti-pro=oxidant status of a lipoprotein particle. The dynamic interactions occurring amongst lipoproteins, their constituents and the remaining antioxidants and prooxidants in the circulation are likely to be very complex. In vitro, $\alpha$-tocopherol associated with low density lipoprotein has been shown to exchange intermolecularly with estimated half-lifes of 20-70 min (9). This study did not attempt to assess the associated lipid peroxide concentrations although this determination may aid in the identification of excess oxidative modification of low density lipoprotein in patients with coronary artery disease.

It is important to note that the association of vitamin $E$ with reduced risk of cardiac disease is strengthened by supplementation of the usual dietary intake (10). In addition, there is evidence that this supplementation must exceed a threshold before protective effects are achieved $(1,11)$. The data obtained here on a group of men not taking vitamin $\mathrm{E}$ supplements support this notation by failing to exhibit significantly higher $\alpha$-tocopherol concentrations or ratios to lipid concentrations in the absence of coronary artery disease. We could not ascertain the effect of dietary vitamin $\mathrm{E}$ on this association and, thus, it cannot be ruled out. The confirmation of the utility of measurement of this antioxidant in serum or plasma will probably require further prospective evaluation of patients receiving significant vitamin $\mathrm{E}$ supplementation, in a diet-controlled situation.

In conclusion, this study demonstrates that $\alpha$-tocopherol concentrations may be actually increased in patients 
with coronary artery disease due to its association with increased concentrations of lipoproteins. This study also confirmed the relevance of an increased total cholesterol concentration to the presence of coronary artery disease when other clinical factors are controlled. Apolipoprotein B and low density lipoprotein-cholesterol were also increased significantly in patients with coronary artery disease. The remaining risk factors analyzed did not

\section{References}

1. Esterbauer, H., Gebicki, J., Puhl, H. \& Jurgens, G. (1992) The role of lipid peroxidation and antioxidants in oxidative modification of LDL. Free Rad. Biol. Med. 13, 341-390.

2. Stringer, M. D., Gorog, P. G., Freeman, A. \& Kakker, V. V. (1989) Lipid peroxides and atherosclerosis. Br. J. Med. 298, 281-284.

3. Warnick, G. R., Benderson, J. \& Albers, J. J. (1982) Dextran sulfate- $\mathrm{Mg}^{2+}$ precipitation procedure for quantitation of high density lipoprotein cholesterol. Clin. Chem. 28, 1379-1388.

4. Warnick, G. R., Benderson, J. \& Albers, J. J. (1982) Quantitation of high-density-lipoprotein subclasses after separation by dextran sulfate and $\mathrm{Mg}^{2+}$ precipitation. Clin. Chem. 28,1574

5. Belcher, J. D., McNamara, J. R., Grinstead, G. F., Rifai, N., Wornick, G. R., Bacharik, P. \& Frantz, I. (1991) Measurement of low density lipoprotein cholesterol concentration. In: $\mathrm{Me}$ thods for Clinical Laboratory Measurement of Lipid and Lipoprotein Risk Factors (Rifai, N. \& Warnick, G. S. R., eds.) pp. 77-83, AACC Press, Washington, D. C.

6. Zar, J. H. (1984) Biostatistical Analysis, 2nd edn., pp. 79335, Prentice-Hall Inc., Englewood Cliffs, New Jersey.

7. Engelman, L. (1985) Stepwise Logistic Regression. In: BMDP Statistical Software Manual (Dixon, W. J., ed.) pp. 330-344, University of California Press, Berkeley, California. achieve significance and their relevance could not be validated. Expressing vitamin $\mathrm{E}$ as a mass ratio eliminated the significance of the difference observed between groups. Therefore, although oxidative modification of low density lipoprotein may be important in the development of atheroslcerosis the measurement of total $\alpha$ tocopherol in serum does not provide useful information regarding its potential role as a preventive antioxidant.

8. Gey, K. F., Puska, P., Jordan, P. \& Moser, U. K. (1991) Inverse correlation between plasma vitamin $\mathrm{E}$ and mortality from ischemic heart disease in cross-cultural epidemiology. Am. J. Clin. Nutr. 53, 326s-334s.

9. Massay, J. B. (1984) Kinetics of transfer of $\alpha$-tocopherol between model and native plasma lipoproteins. Biochim. Biophys. Acta 793, 387-432.

10. Rimm, E. B., Stampfer, M. J., Ascherio, A., Giovannucci, E., Colditz, G. A. \& Willett, W. C. (1993) Vitamin E consumption and the risk of coronary heart disease in men. N. Engl. J. Med. $328,1450-1456$.

11. Dieber-Rotheneder, M., Puhl, M., Waeg, G., Striegl, G. \& Esterbauer, H. (1991) Effect of oral supplementation with D- $\alpha$ tocopherol on the vitamin $E$ content of human low density lipoproteins and resistance to oxidation. J. Lipid Res. 32, $1325-1332$.

Prof. Dr. Frederick Van Lente

Section of Biochemistry

Division of Pathology and

Laboratory Medicine

Cleveland Clinic Foundation

9500 Euclid Avenue

Cleveland, Ohio, 44195

U.S.A. 
